\title{
Measurement of charged pion double spin asymmetries at midrapidity in longitudinally polarized $p+p$ collisions at $\sqrt{s}=510 \mathrm{GeV}$
}

U. Acharya,${ }^{20}$ A. Adare, ${ }^{11}$ C. Aidala, ${ }^{43}$ N. N. Ajitanand, ${ }^{62,{ }^{*}}$ Y. Akiba,${ }^{57,58, \dagger}$ R. Akimoto, ${ }^{10}$ M. Alfred, ${ }^{23}$ N. Apadula,,${ }^{28,63}$ Y. Aramaki,${ }^{57}$ H. Asano, ${ }^{35,57}$ E. T. Atomssa, ${ }^{63}$ T. C. Awes,${ }^{52}$ B. Azmoun, ${ }^{7}$ V. Babintsev, ${ }^{24}$ M. Bai ${ }^{6}$ N. S. Bandara, ${ }^{42}$ B. Bannier, ${ }^{63}$ K. N. Barish, ${ }^{8}$ S. Bathe, ${ }^{5,58}$ A. Bazilevsky, ${ }^{7}$ M. Beaumier, ${ }^{8}$ S. Beckman, ${ }^{11}$ R. Belmont, ${ }^{11,43,50}$ A. Berdnikov, ${ }^{60}$ Y. Berdnikov ${ }^{60}$ D. Black, ${ }^{8}$ J. S. Bok, ${ }^{49}$ K. Boyle, ${ }^{58}$ M. L. Brooks, ${ }^{38}$ J. Bryslawskyj ${ }^{5,8}$ H. Buesching, ${ }^{7}$ V. Bumazhnov, ${ }^{24}$ S. Campbell, ${ }^{12,28}$ V. Canoa Roman, ${ }^{63}$ C.-H. Chen, ${ }^{58}$ C. Y. Chi, ${ }^{12}$ M. Chiu, ${ }^{7}$ I. J. Choi, ${ }^{25}$ J. B. Choi, ${ }^{30}$ T. Chujo, ${ }^{66}$ Z. Citron, ${ }^{68}$ M. Connors, ${ }^{20,58}$ M. Csanád, ${ }^{15}$ T. Csörgő, ${ }^{16,69}$ T. W. Danley, ${ }^{51}$ A. Datta, ${ }^{48}$ M. S. Daugherity, ${ }^{1}$ G. David,,${ }^{7,14,63}$ K. DeBlasio, ${ }^{48}$

K. Dehmelt, ${ }^{63}$ A. Denisov, ${ }^{24}$ A. Deshpande, ${ }^{7,58,63}$ E. J. Desmond, ${ }^{7}$ L. Ding,${ }^{28}$ A. Dion, ${ }^{63}$ J. H. Do,${ }^{70}$ A. Drees, ${ }^{63}$

K. A. Drees ${ }^{6}$ J. M. Durham,${ }^{38}$ A. Durum,${ }^{24}$ A. Enokizono, ${ }^{57,59}$ H. En'yo, ${ }^{57}$ R. Esha ${ }^{63}$ S. Esumi,${ }^{66}$ B. Fadem,${ }^{44}$ W. Fan, ${ }^{63}$

N. Feege, ${ }^{63}$ D. E. Fields, ${ }^{48}$ M. Finger, ${ }^{9}$ M. Finger, Jr., ${ }^{9}$ D. Fitzgerald, ${ }^{43}$ S. L. Fokin, ${ }^{34}$ J. E. Frantz, ${ }^{51}$ A. Franz, ${ }^{7}$

A. D. Frawley, ${ }_{19}$ C. Gal, ${ }^{63}$ P. Gallus, ${ }^{13}$ E. A. Gamez,${ }^{43}$ P. Garg, ${ }^{3,63}$ H. Ge ${ }^{63}$ F. Giordano, ${ }^{25}$ A. Glenn,${ }^{37}$ Y. Goto, ${ }^{57,58}$ N. Grau ${ }^{2}$ S. V. Greene, ${ }^{67}$ M. Grosse Perdekamp,${ }^{25}$ Y. Gu ${ }^{62}$ T. Gunji ${ }^{10}$ H. Guragain, ${ }^{20}$ T. Hachiya ${ }^{46,57,58}$ J. S. Haggerty, ${ }^{7}$ K. I. Hahn, ${ }^{17}$ H. Hamagaki, ${ }^{10}$ S. Y. Han, ${ }^{17,33,57}$ J. Hanks, ${ }^{63}$ S. Hasegawa, ${ }^{29}$ T. O. S. Haseler, ${ }^{20}$ X. He, ${ }^{20}$ T. K. Hemmick, ${ }^{63}$ J. C. Hill, ${ }^{28}$ K. Hill, ${ }^{11}$ A. Hodges, ${ }^{20}$ R. S. Hollis, ${ }^{8}$ K. Homma, ${ }^{22}$ B. Hong, ${ }^{33}$ T. Hoshino, ${ }^{22}$ J. Huang, ${ }^{7,38}$ S. Huang,${ }^{67}$ Y. Ikeda,${ }^{57}$ K. Imai ${ }^{29}$ Y. Imazu, ${ }^{57}$ M. Inaba, ${ }^{66}$ A. Iordanova, ${ }^{8}$ D. Isenhower, ${ }^{4}$ S. Ishimaru, ${ }^{46}$ D. Ivanishchev ${ }^{55}$ B. V. Jacak, ${ }^{63}$ S. J. Jeon, ${ }^{45}$ M. Jezghani, ${ }^{20}$ Z. Ji, ${ }^{63}$ J. Jia, ${ }^{7,62}$ X. Jiang, ${ }^{38}$ B. M. Johnson $\odot,{ }^{7,20}$ E. Joo, ${ }^{33}$ K. S. Joo, ${ }^{45}$ D. Jouan, ${ }^{53}$ D. S. Jumper, ${ }^{25}$

J. H. Kang, ${ }^{70}$ J. S. Kang, ${ }^{21}$ D. Kawall, ${ }^{42}$ A. V. Kazantsev, ${ }^{34}$ J. A. Key, ${ }^{48}$ V. Khachatryan ${ }^{63}$ A. Khanzadeev, ${ }^{55}$

A. Khatiwada, ${ }^{38}$ K. Kihara, ${ }^{66}$ C. Kim ${ }^{33}$ D. H. Kim,${ }^{17}$ D. J. Kim, ${ }^{31}$ E.-J. Kim, ${ }^{30}$ H.-J. Kim, ${ }^{70}$ M. Kim, ${ }^{57,61}$ Y. K. Kim ${ }^{21}$ D. Kincses, ${ }^{15}$ E. Kistenev, ${ }^{7}$ J. Klatsky, ${ }^{19}$ D. Kleinjan, ${ }^{8}$ P. Kline,${ }^{63}$ T. Koblesky, ${ }_{11}^{11}$ M. Kofarago, ${ }_{15,69}$ J. Koster, ${ }^{58}$ D. Kotov,${ }^{55,60}$

B. Kurgyis ${ }^{15}$ K. Kurita, ${ }^{59}$ M. Kurosawa ${ }^{57,58}$ Y. Kwon, ${ }^{70}$ R. Lacey ${ }^{62}$ J. G. Lajoie ${ }^{28}$ A. Lebedev, ${ }^{28}$ K. B. Lee ${ }^{38}$

S. H. Lee, ${ }^{28,63}$ M. J. Leitch, ${ }^{38}$ M. Leitgab, ${ }^{25}$ Y. H. Leung, ${ }^{63}$ N. A. Lewis, ${ }^{43}$ X. Li, ${ }^{38}$ S. H. Lim, ${ }^{11,38,56,70}$ M. X. Liu, ${ }^{38}$

S. Lökös, ${ }^{15,16}$ D. Lynch, ${ }^{7}$ T. Majoros, ${ }^{14}$ Y. I. Makdisi, ${ }^{6}$ M. Makek,${ }^{68,71}$ A. Manion, ${ }^{63}$ V. I. Manko, ${ }^{34}$ E. Mannel, ${ }^{7}$ M. McCumber ${ }^{38}$ P. L. McGaughey, ${ }^{38}$ D. McGlinchey, ${ }^{1,38}$ C. McKinney, ${ }^{25}$ A. Meles, ${ }^{49}$ M. Mendoza ${ }^{8}{ }^{8}$ B. Meredith, ${ }^{12}$ W. J. Metzger, ${ }^{16}$ Y. Miake, ${ }^{66}$ A. C. Mignerey, ${ }^{41}$ A. J. Miller, ${ }^{1}$ A. Milov, ${ }^{68}$ D. K. Mishra, ${ }^{4}$ J. T. Mitchell, ${ }^{7}$ Iu. Mitrankov, ${ }^{60}$ G. Mitsuka, ${ }^{32,57}$ S. Miyasaka, ${ }^{57,65}$ S. Mizuno, ${ }^{57,66}$ P. Montuenga, ${ }^{25}$ T. Moon, ${ }^{33,57,70}$ D. P. Morrison, ${ }^{7}$ S. I. Morrow, ${ }^{67}$

T. V. Moukhanova, ${ }^{34}$ B. Mulilo, ${ }^{33,57}$ T. Murakami, ${ }^{35,57}$ J. Murata, ${ }^{57,59}$ A. Mwai, ${ }^{62}$ S. Nagamiya, ${ }^{32,57}$ K. Nagashima, ${ }^{22,57}$ J. L. Nagle, ${ }^{11}$ M. I. Nagy, ${ }^{15}$ I. Nakagawa, ${ }^{57,58}$ H. Nakagomi, ${ }^{57,66}$ K. Nakano, ${ }^{57,65}$ C. Nattrass, ${ }^{64}$ S. Nelson, ${ }^{18}$

P. K. Netrakanti, ${ }^{4}$ M. Nihashi, ${ }^{22,57}$ T. Niida, ${ }^{66}$ R. Nishitani, ${ }^{46}$ R. Nouicer, ${ }^{7,58}$ T. Novák, ${ }^{16,69}$ N. Novitzky, ${ }^{31,63,66}$

A. S. Nyanin, ${ }^{34}$ E. O’Brien, ${ }^{7}$ C. A. Ogilvie, ${ }^{28}$ J. D. Orjuela Koop, ${ }^{11}$ J. D. Osborn, ${ }^{43}$ A. Oskarsson,${ }^{39}$ K. Ozawa, ${ }^{32,66}$ R. Pak, V. Pantuev, ${ }^{26}$ V. Papavassiliou, ${ }^{49}$ S. Park, ${ }^{57,61,63}$ S. F. Pate, ${ }^{49}$ L. Patel, ${ }^{20}$ M. Patel, ${ }^{28}$ J.-C. Peng, ${ }_{7,63}$ W. Peng, ${ }^{67}$

D. V. Perepelitsa, ${ }^{711,12}$ G. D. N. Perera, ${ }^{49}$ D. Yu. Peressounko, ${ }^{34}$ C. E. PerezLara,${ }^{63}$ J. Perry, ${ }^{28}$ R. Petti, ${ }^{7,63}$ C. Pinkenburg, ${ }^{7}$

R. Pinson, ${ }^{1}$ R. P. Pisani, ${ }^{7}$ M. Potekhin, ${ }^{7}$ A. Pun,${ }^{51}$ M. L. Purschke, ${ }^{7}$ P. V. Radzevich, ${ }^{60}$ J. Rak ${ }^{31}$ N. Ramasubramanian ${ }^{63}$ I. Ravinovich, ${ }^{68}$ K. F. Read, ${ }^{52,64}$ D. Reynolds, ${ }^{62}$ V. Riabov, ${ }^{47,55}$ Y. Riabov,${ }^{55,60}$ D. Richford, ${ }^{5}$ T. Rinn, ${ }^{25,28}$ N. Riveli, ${ }^{51}$

D. Roach, ${ }^{67}$ S. D. Rolnick, ${ }^{8}$ M. Rosati, ${ }^{28}$ Z. Rowan, ${ }^{5}$ J. G. Rubin, ${ }^{43}$ J. Runchey, ${ }^{28}$ N. Saito, ${ }^{32}$ T. Sakaguchi, ${ }^{7}$ H. Sako, ${ }^{29}$ V. Samsonov, ${ }^{47,55}$ M. Sarsour, ${ }^{20}$ S. Sato, ${ }^{29}{ }^{8}$ S. Sawada, ${ }^{32}$ C. Y. Scarlett, ${ }^{18}$ B. Schaefer,${ }_{67}^{67}$ B. K. Schmoll, ${ }_{57,65}$ K. Sedgwick, ${ }^{8}$ J. Seele,${ }^{58}$ R. Seidl, ${ }^{57,58}$ A. Sen, ${ }^{28,64}$ R. Seto, ${ }^{8}$ P. Sett, ${ }^{4}$ A. Sexton, ${ }^{41}$ D. Sharma,${ }^{63}$ I. Shein, ${ }^{24}$ T.-A. Shibata, ${ }^{57,65}$ K. Shigaki, ${ }^{22}$ M. Shimomura, ${ }^{28,46}$ P. Shukla, ${ }^{4}$ A. Sickles, ${ }^{7,25}$ C. L. Silva, ${ }^{38}$ D. Silvermyr, ${ }^{39,52}$ B. K. Singh, ${ }^{3}$ C. P. Singh, ${ }^{3}$ V. Singh, ${ }^{3}$

M. Slunečka, ${ }^{9}$ K. L. Smith, ${ }^{19}$ R. A. Soltz, ${ }^{37}$ W. E. Sondheim, ${ }^{38}$ S. P. Sorensen, ${ }^{64}$ I. V. Sourikova, ${ }^{7}$ P. W. Stankus, ${ }^{52}$

M. Stepanov, ${ }^{42}$ S. P. Stoll, ${ }^{7}$ T. Sugitate, ${ }^{22}$ A. Sukhanov, ${ }^{7}$ T. Sumita,${ }^{57}$ J. Sun, ${ }^{63}$ X. Sun ${ }^{20}$ Z. Sun, ${ }^{14}$ S. Suzuki, ${ }^{46}$ J. Sziklai, ${ }^{69}$ A. Takahara, ${ }^{10}$ A. Taketani, ${ }^{77,58}$ K. Tanida ${ }^{29,58,61}$ M. J. Tannenbaum, ${ }^{7}$ S. Tarafdar, ${ }^{67,68}$ A. Taranenko, ${ }^{47,62}$ R. Tieulent, ${ }^{40}$ A. Timilsina, ${ }^{28}$ T. Todoroki, ${ }^{57,58,66}$ M. Tomášek,${ }^{13}$ H. Toriii, ${ }^{10}$ M. Towell,,${ }^{1}$ R. Towell, ${ }^{1}$ R. S. Towell ${ }^{1}$ I. Tserruya,${ }^{68}$

Y. Ueda, ${ }^{22}$ B. Ujvari, ${ }^{14}$ H. W. van Hecke, ${ }^{38}$ M. Vargyas, ${ }^{15,69}$ J. Velkovska, ${ }^{67}$ M. Virius ${ }^{13}$ V. Vrba, ${ }^{13,27}$ E. Vznuzdaev, ${ }^{55}$ X. R. Wang ${ }^{49,58}$ Z. Wang, ${ }^{5}$ D. Watanabe, ${ }^{22}$ Y. Watanabe, ${ }^{57,58}$ Y. S. Watanabe, ${ }^{10,32}$ F. Wei, ${ }^{49}$ S. Whitaker,${ }^{28}$ S. Wolin, ${ }^{25}$ C. P. Wong, ${ }^{20,38}$ C. L. Woody, ${ }^{7}$ Y. Wu, ${ }^{8}$ M. Wysocki ${ }^{52}$ B. Xia, ${ }^{51}$ Q. Xu,${ }^{67}$ L. Xue, ${ }^{20}$ S. Yalcin, ${ }^{63}$ Y. L. Yamaguchi, ${ }^{10,58,63}$ A. Yanovich, ${ }^{24}$ J. H. Yoo, ${ }^{33,58}$ I. Yoon, ${ }^{61}$ I. Younus,${ }^{36}$ H. Yu, ${ }^{49,54}$ I. E. Yushmanov, ${ }^{34}$ W. A. Zajc, ${ }^{12}$ A. Zelenski, ${ }^{6}$ Y. Zhai, ${ }^{28}$ S. Zharko, ${ }^{60}$ and L. Zou ${ }^{8}$

(PHENIX Collaboration)

${ }^{1}$ Abilene Christian University, Abilene, Texas 79699, USA

${ }^{2}$ Department of Physics, Augustana University, Sioux Falls, South Dakota 57197, USA 


\footnotetext{
${ }^{3}$ Department of Physics, Banaras Hindu University, Varanasi 221005, India

${ }^{4}$ Bhabha Atomic Research Centre, Bombay 400 085, India

${ }^{5}$ Baruch College, City University of New York, New York, New York 10010, USA

${ }^{6}$ Collider-Accelerator Department, Brookhaven National Laboratory, Upton, New York 11973-5000, USA

${ }^{7}$ Physics Department, Brookhaven National Laboratory, Upton, New York 11973-5000, USA

${ }^{8}$ University of California-Riverside, Riverside, California 92521, USA

${ }^{9}$ Charles University, Ovocný trh 5, Praha 1, 116 36, Prague, Czech Republic

${ }^{10}$ Center for Nuclear Study, Graduate School of Science, University of Tokyo, 7-3-1 Hongo, Bunkyo, Tokyo 113-0033, Japan

${ }^{11}$ University of Colorado, Boulder, Colorado 80309, USA

${ }^{12}$ Columbia University, New York, New York 10027, USA, and Nevis Laboratories, Irvington, New York 10533, USA

${ }^{13}$ Czech Technical University, Zikova 4, 16636 Prague 6, Czech Republic

${ }^{14}$ Debrecen University, H-4010 Debrecen, Egyetem tér 1, Hungary

${ }^{15}$ ELTE, Eötvös Loránd University, H-1117 Budapest, Pázmány P. s. 1/A, Hungary

${ }^{16}$ Eszterházy Károly University, Károly Róbert Campus, H-3200 Gyöngyös, Mátrai út 36, Hungary

${ }^{17}$ Ewha Womans University, Seoul 120-750, Korea

${ }^{18}$ Florida A\&M University, Tallahassee, Florida 32307, USA

${ }^{19}$ Florida State University, Tallahassee, Florida 32306, USA

${ }^{20}$ Georgia State University, Atlanta, Georgia 30303, USA

${ }^{21}$ Hanyang University, Seoul 133-792, Korea

${ }^{22}$ Hiroshima University, Kagamiyama, Higashi-Hiroshima 739-8526, Japan

${ }^{23}$ Department of Physics and Astronomy, Howard University, Washington, D.C. 20059, USA

${ }^{24}$ IHEP Protvino, State Research Center of Russian Federation,

Institute for High Energy Physics, Protvino, 142281, Russia

${ }^{25}$ University of Illinois at Urbana-Champaign, Urbana, Illinois 61801, USA

${ }^{26}$ Institute for Nuclear Research of the Russian Academy of Sciences,

prospekt 60-letiya Oktyabrya 7a, Moscow 117312, Russia

${ }^{27}$ Institute of Physics, Academy of Sciences of the Czech Republic, Na Slovance 2, 18221 Prague 8, Czech Republic

${ }^{28}$ Iowa State University, Ames, Iowa 50011, USA

${ }^{29}$ Advanced Science Research Center, Japan Atomic Energy Agency, 2-4 Shirakata Shirane, Tokai-mura, Naka-gun, Ibaraki-ken 319-1195, Japan

${ }^{30}$ Jeonbuk National University, Jeonju, 54896, Korea

${ }^{31}$ Helsinki Institute of Physics and University of Jyväskylä, P.O. Box 35, FI-40014 Jyväskylä, Finland

${ }^{32}$ KEK, High Energy Accelerator Research Organization, Tsukuba, Ibaraki 305-0801, Japan

${ }^{33}$ Korea University, Seoul 02841, Korea

${ }^{34}$ National Research Center “Kurchatov Institute," Moscow, 123098 Russia

${ }^{35}$ Kyoto University, Kyoto 606-8502, Japan

${ }^{36}$ Physics Department, Lahore University of Management Sciences, Lahore 54792, Pakistan

${ }^{37}$ Lawrence Livermore National Laboratory, Livermore, California 94550, USA

${ }^{38}$ Los Alamos National Laboratory, Los Alamos, New Mexico 87545, USA

${ }^{39}$ Department of Physics, Lund University, Box 118, SE-221 00 Lund, Sweden

${ }^{40}$ IPNL, CNRS/IN2P3, Université Lyon, Université Lyon 1, F-69622, Villeurbanne, France

${ }^{41}$ University of Maryland, College Park, Maryland 20742, USA

${ }^{42}$ Department of Physics, University of Massachusetts, Amherst, Massachusetts 01003-9337, USA

${ }^{43}$ Department of Physics, University of Michigan, Ann Arbor, Michigan 48109-1040, USA

${ }^{44}$ Muhlenberg College, Allentown, Pennsylvania 18104-5586, USA

${ }^{45}$ Myongji University, Yongin, Kyonggido 449-728, Korea

${ }^{46}$ Nara Women's University, Kita-uoya Nishi-machi Nara 630-8506, Japan

${ }^{47}$ National Research Nuclear University, MEPhI, Moscow Engineering Physics Institute, Moscow, 115409, Russia

${ }^{48}$ University of New Mexico, Albuquerque, New Mexico 87131, USA

${ }^{49}$ New Mexico State University, Las Cruces, New Mexico 88003, USA

${ }^{50}$ Physics and Astronomy Department, University of North Carolina at Greensboro, Greensboro, North Carolina 27412, USA

${ }^{51}$ Department of Physics and Astronomy, Ohio University, Athens, Ohio 45701, USA

${ }^{52}$ Oak Ridge National Laboratory, Oak Ridge, Tennessee 37831, USA

${ }^{53}$ IPN-Orsay, Université Paris-Sud, CNRS/IN2P3, Université Paris-Saclay, BP1, F-91406, Orsay, France

${ }^{54}$ Peking University, Beijing 100871, People's Republic of China
} 


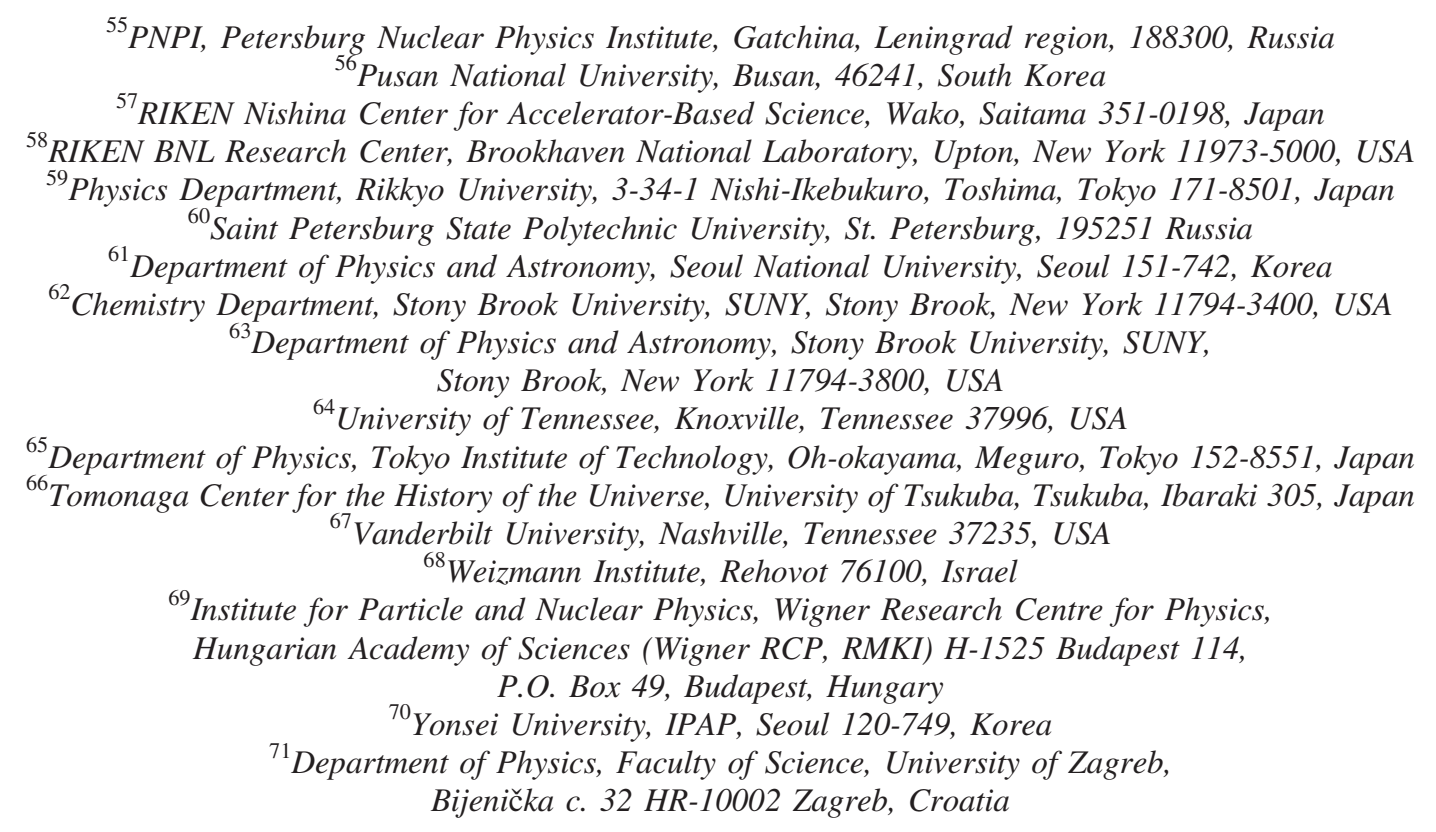

(Received 7 April 2020; accepted 13 July 2020; published 5 August 2020)

\begin{abstract}
The PHENIX experiment at the Relativistic Heavy Ion Collider has measured the longitudinal double spin asymmetries, $A_{L L}$, for charged pions at midrapidity $(|\eta|<0.35)$ in longitudinally polarized $p+p$ collisions at $\sqrt{s}=510 \mathrm{GeV}$. These measurements are sensitive to the gluon spin contribution to the total spin of the proton in the parton momentum fraction $x$ range between 0.04 and 0.09 . One can infer the sign of the gluon polarization from the ordering of pion asymmetries with charge alone. The asymmetries are found to be consistent with global quantum-chromodynamics fits of deep-inelastic scattering and data at $\sqrt{s}=200 \mathrm{GeV}$, which show a nonzero positive contribution of gluon spin to the proton spin.
\end{abstract}

DOI: 10.1103/PhysRevD.102.032001

\section{INTRODUCTION}

The spin of the proton is known to be $\hbar / 2$, yet its decomposition in terms of its constituents, quarks and gluons, is not very well known. Initially, the fixed-target deep-inelastic scattering (DIS) experiments measured the polarized structure function, $g_{1}\left(x, Q^{2}\right)$, where $x$ is the parton momentum fraction of the proton and $Q^{2}$ is the momentum transfer squared, enabling the reconstruction of the quark spin contributions, $\Delta \Sigma\left(x, Q^{2}\right)$, with the help of weak and hyperon decay constants. Early measurements found this contribution to be substantially smaller than expected [1], leading to the so-called spin crisis. In addition to the quark spins, gluon spins as well as the constituents' orbital angular momenta can contribute to the spin sum rule

\footnotetext{
*Deceased.

${ }^{\dagger}$ PHENIX spokesperson: akiba@rcf.rhic.bnl.gov
}

Published by the American Physical Society under the terms of the Creative Commons Attribution 4.0 International license. Further distribution of this work must maintain attribution to the author(s) and the published article's title, journal citation, and DOI. Funded by SCOAP ${ }^{3}$.
[2]. Because DIS at low to moderate energies essentially couples through the electromagnetic interaction, it is most sensitive to the quark spin contributions and the gluon spin only enters via scaling violations.

In contrast, in polarized $p+p$ collisions, for example at the Relativistic Heavy Ion Collider (RHIC), the dominant hard interaction happens via the strong interaction. Therefore, for midrapidity $(|\eta|<0.35)$ hadronic or jet final states with small to intermediate energies, quark-gluon and gluon-gluon interactions are the dominant processes. Consequently, longitudinal-double-spin asymmetries, $A_{L L}$, are sensitive to the gluon-spin contribution to the proton, $\Delta g\left(x, Q^{2}\right)$. The RHIC jet [3] and neutral pion asymmetry measurements [4] at a center-of-mass energy, $\sqrt{s}$, of $200 \mathrm{GeV}$ resulted in the first indication of a nonzero gluon-spin contribution to the nucleon spin when the jet and neutral-pion data was analyzed together with the DIS and semi-inclusive DIS results in a global analysis [5,6]. Subsequently, various measurements at a higher collision energy of $510 \mathrm{GeV}$ have confirmed this nonzero gluon polarization [7-10] and those combined with results at $\sqrt{s}=200 \mathrm{GeV}$ [11] have extended the parton momentum fraction $x$ coverage to lower values of approximately $10^{-3}$. 
While the global fits clearly prefer a positive gluon polarization in the probed $x$ range, another direct experimental confirmation would be helpful. The addition of charged pion asymmetries with the help of different fragmentation of up and down quarks [12] into $\pi^{ \pm}$provides this possibility. Because up and down quark polarizations are reasonably well known, the ordering of the positive, neutral and negative pion asymmetries immediately informs about the sign of the gluon spin. A positive gluon spin, coupled with the positive up quark polarization and negative down quark polarization would result in $\pi^{+}$ asymmetries to be the largest, followed by $\pi^{0}$ and, then, $\pi^{-}$. The charge-separated pion asymmetry results at $\sqrt{s}=200 \mathrm{GeV}$ have already been published [13].

In this paper, we report the charged pion longitudinal double spin asymmetries at $\sqrt{s}=510 \mathrm{GeV}$ that were extracted by the PHENIX experiment at midrapidity. The paper is organized as follows. In Sec. II, the PHENIX experiment and the detector components relevant for this result are described. In Sec. III, the analysis procedure for extracted charged pions and their double spin asymmetries at midrapidity is discussed. In Sec. IV, the results are presented. The summary is given in Sec. V.

\section{EXPERIMENTAL SETUP}

In 2013, the PHENIX experiment at RHIC collected data from longitudinally polarized $p+p$ collisions at $\sqrt{s}=510 \mathrm{GeV}$ with an average polarization of 0.55 and 0.56 for the clockwise (blue) and counterclockwise (yellow) beams, respectively. An integrated luminosity of $108 \mathrm{pb}^{-1}$ was sampled for charged-pion asymmetry measurements at midrapidity.

The PHENIX detector is described in detail in Ref. [14]. Each of two nearly back-to-back arms of the central spectrometer covers a rapidity range $|\eta|<0.35$ and an azimuthal range of $\Delta \phi=\frac{\pi}{2}$. The PHENIX detector elements used in this analysis include the drift chambers (DC), the pad chambers (PC), the ring imaging Čerenkov (RICH) detector and the electromagnetic calorimeters (EMCal). The $\mathrm{RICH}$, filled with $\mathrm{CO}_{2}$ gas radiator, is used for charged-pion identification. The EMCal comprises two different types of calorimeters. Six sectors are constructed with lead-scintillator $(\mathrm{PbSc})$ towers in sampling configuration with depth of 0.85 interaction lengths. Two sectors are made of lead-glass towers with a depth of 1.05 nuclear interaction lengths. Because the events sampled for this analysis are triggered via energy deposit thresholds, only the fraction of pions that shower in the EMCal are available. Analysis is limited to the PbSc-triggered events, because the higher-energy thresholds result in lower background fractions than in the lead-glass towers. Charged particle tracks are reconstructed with the DC and PC tracking system. These detectors also provide the momentum information of the tracks. A match between a projected track onto the EMCal and the location of deposited energy is required to veto charged tracks with mis-reconstructed momenta. The silicon-vertex detector surrounds the beam pipe with layers at nominal radii $2.6,5.1,11.8,16.7 \mathrm{~cm}$ with an acceptance of $|\eta|<1$ and $\Delta \phi=0.8 \pi$. The total material budget is 0.13 radiation lengths and the detector was not in operation in 2013. This created a large source of electron background from conversions of direct and decay photons.

Additionally, two sets of 64 quartz-crystal radiators attached to photomultipliers located at $z$ positions of $\pm 144 \mathrm{~cm}$ and rapidities between 3.1 to 3.9 were used to trigger hard collision events and to select events within $\pm 30 \mathrm{~cm}$ of the collision vertex in the asymmetry analysis. These beam-beam counters and the zero-degree calorimeters were used together to evaluate the luminosities seen by the PHENIX detector. The zero-degree calorimeters, comprising three sections of a hadronic calorimeter located at $\pm 18 \mathrm{~m}$ from the PHENIX interaction point, are also used to monitor the polarization orientation and confirm that the polarization direction of the beams has been rotated to the longitudinal direction.

\section{ANALYSIS PROCEDURE}

\section{A. Dataset and triggers}

The 2013 detector configuration was similar to the published results at $\sqrt{s}=200 \mathrm{GeV}$ [13] in 2009, except that the hadron-blind detector was no longer installed. Due to the higher collision energy and collision rates in 2013, the energy thresholds of the EMCal triggers were increased by a factor of $\approx 2-3$ compared to in 2009 and events were triggered by particles leaving at least $2.2,3.7,4.7$ or $5.6 \mathrm{GeV}$ energy deposits in the EMCal for the various trigger types. The lower energy threshold triggers were prescaled such that only a fraction of events satisfying the trigger requirements was recorded. A logical OR of all these triggers (i.e. if any trigger condition was met) was used for the transverse momentum bins in the range $5 \mathrm{GeV} / c<p_{T}<11 \mathrm{GeV} / c$, where the less prescaled higher threshold triggers are dominant. To minimize the background contribution for the highest transverse momentum bin $\left(11 \mathrm{GeV} / c<p_{T}<15 \mathrm{GeV} / c\right)$, the $2.2 \mathrm{GeV}$ threshold trigger was not used. The trigger efficiency curves as a function of transverse momentum with energy threshold of $3.7 \mathrm{GeV}$ for the PbSc are displayed in Fig. 1 for $\pi^{ \pm}$ candidates where also a preselection cut on the ratio between cluster energy to reconstructed momentum $(E / p$, to be described in detail below) was already applied. High $p_{T}$ charged pions punch through the EMCal with approximately a $50 \%$ chance, depositing only a small fraction of their energy corresponding to the minimum-ionizing particles (MIPs) at $\approx 0.3 \mathrm{GeV}$ due to their low probability of nuclear interactions in the detector. The preselection cuts for $\pi^{ \pm}$are blind to the MIP interactions and consequently result 


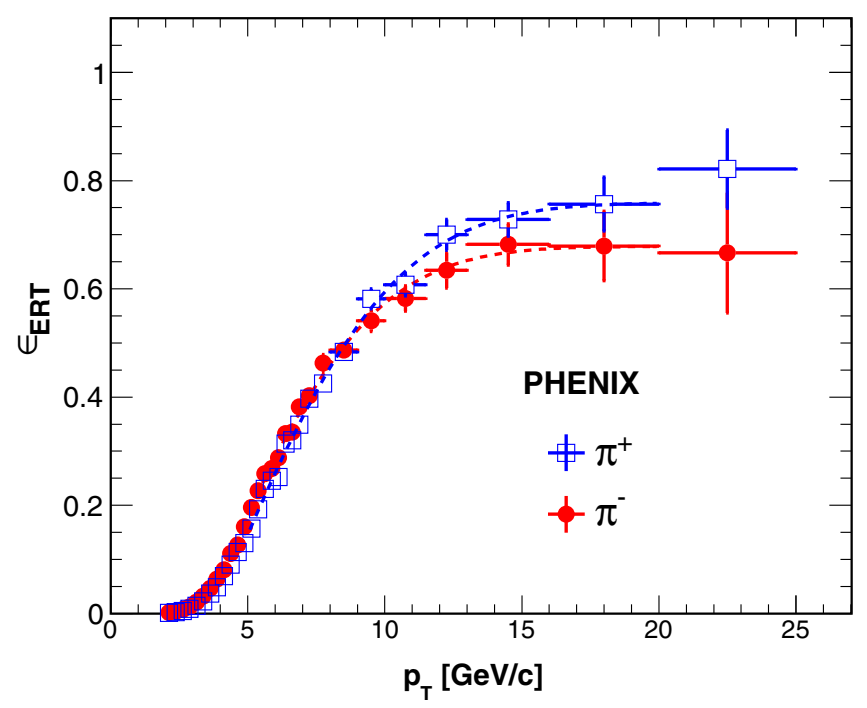

FIG. 1. Trigger efficiency curves of the EMCal-RICH trigger for positively charged (open [blue] squares) and negatively charged (closed [red] circles) pion candidates in the PbSc as a function of the transverse momentum of the track. The energy threshold of the trigger was at $3.7 \mathrm{GeV}$. Note that a cut on the ratio between cluster energy to reconstructed momentum $(E / p)$ was applied in preselection of the $\pi^{ \pm}$sample. The charge difference seen at higher $p_{T}$ originates from the momentum reconstruction which could not be perfectly calibrated in the high rate conditions of the 2013 data taking period.

in higher trigger efficiencies than for the case where all types of interactions are taken into account. Nonetheless, this analysis does not include MIPs, and the approach properly takes into account the $p_{T}$ dependence of trigger efficiency after applying preselection cuts.

\section{B. Charged pion identification and background estimation}

In addition to the trigger, a matching track in the drift chamber is required to be pointing to the EMCal tower that fired the trigger. The transverse momentum of the particle is determined by the bending of the track in the magnetic field before the DC. In addition, the reconstructed tracks are required to fire more than one photomultiplier by Čerenkov light in the RICH. The threshold for pions is around $4.9 \mathrm{GeV}$ and until the kaon threshold of $17.3 \mathrm{GeV}$ is reached the RICH fires only for pions and electrons (muons are not dominant and are already eliminated by the energy cut from the high energy threshold of trigger). To remove electrons as well as accidental track-EMCal cluster coincidences, the ratio between cluster energy and track momentum $(E / p)$ is required to be larger than 0.2 and smaller than 0.8 , taking into account that most pions do not deposit all their energy in the electromagnetic calorimeter in contrast to electrons.

For the further rejection of electron background from the charged pion candidates, the probability that a cluster has developed via electromagnetic shower processes (shower shape) was determined from fitting the well understood electromagnetic shower shape in the EMCal to the cluster in question. The shower shape probability was required to be less than 0.1. The succession of the selection criteria on the raw charged particle spectra can be seen in Fig. 2. A clear bump can be seen once the momentum is large enough for pions to emit Čerenkov light. The contribution at momenta below the bump indicates remaining electrons and other accidental coincidences. After applying electron rejection cuts, their contributions are substantially reduced $(\approx 0.01-0.085)$. The remaining background in the higher transverse momentum range is studied with full MC simulations using PYTHIA [15] as event generator and GEANT3 [16] for the detector description. Figure 3 shows that at low transverse momenta below $5 \mathrm{GeV} / c$ the distribution is dominated by electrons, accidental pion coincidences, and (to a smaller extent) kaons and protons. At higher transverse momenta, electrons are the dominant background, which is small compared to pion signals until the RICH hit requirement becomes fulfilled by kaons as well. The simulated contributions describe reasonably well both the signaldominated region at higher transverse momenta and the background-dominated region below $5 \mathrm{GeV} / c$.

The relative size of pion signal and electron backgrounds is then further compared with data by studying the full $E / p$ range including the electron peak at ratios around unity where it is quite prominent. Based on this comparison, as seen in Fig. 4, the nonpion background is found to be below a few percent. A Gaussian function for the electron peak and an error function for the pion signal are fit to the $E / p$ distribution in each $p_{T}$ bin. The extracted parameter of the

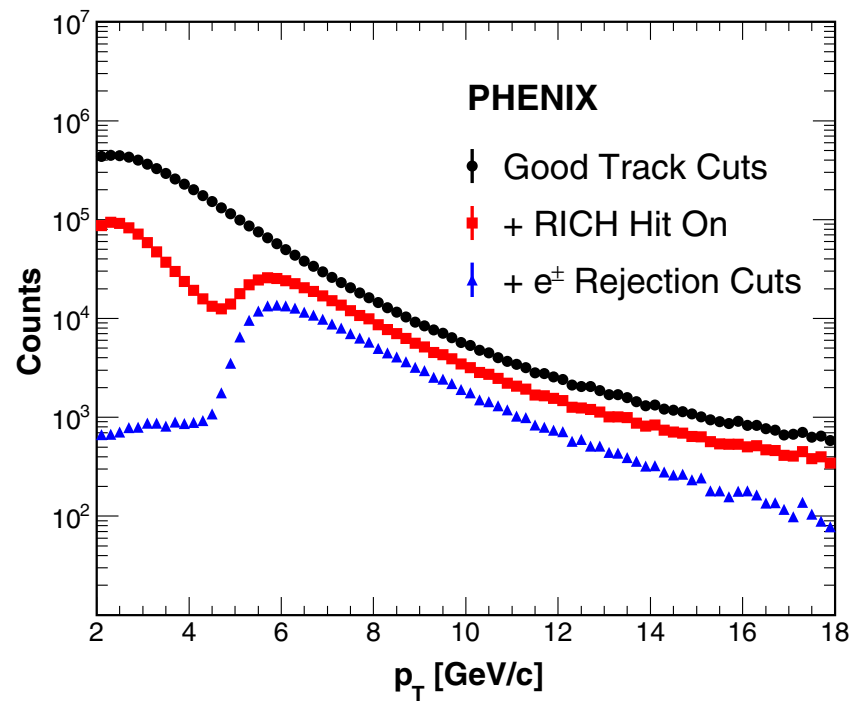

FIG. 2. Pion candidate transverse momentum distributions after successively applying raw track criteria (closed [black] circles), $\mathrm{RICH}$ hit requirement (closed [red] squares) and electron rejection via $E / p$, matching and shower shape (closed [blue] triangles). 


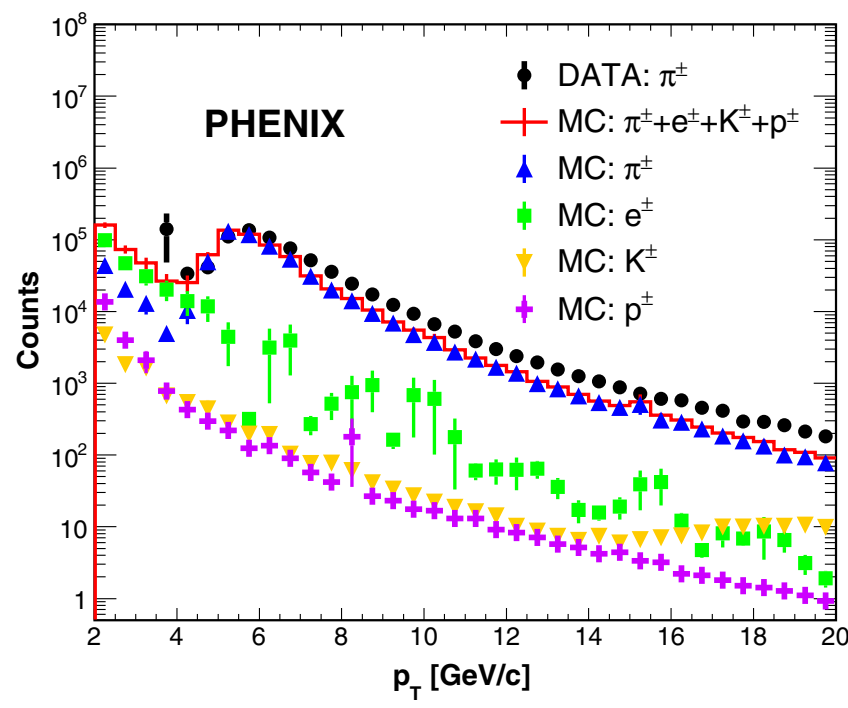

FIG. 3. Comparison of reconstructed particle momentum distributions as a function of the transverse momentum in the data and MC simulations. The pion candidates of the data (closed [black] circles) are corrected for the trigger efficiency. The pion (closed [blue] triangles), electron (closed [green] squares), kaon (closed [yellow] inverted triangles), proton (closed [purple] crosses), and all (histogram [red] lines) contributions of the MC simulation are scaled by the luminosity for apple-to-apple comparison.

Gaussian was used to scale the electron background from the simulation. As the background level was found to be small, the scaling factor was varied by a factor of 2 in the background corrected asymmetries, variation was assigned as systematic uncertainty and the effect of the scale variation found to be small.

\section{Asymmetry analysis}

The selected pions are then separated by a spin pattern, which determines whether the protons collided with the same or opposite helicities. These asymmetries are normalized for the fluctuations in luminosity from the bunch crossings with the same $(++)$ helicity and opposite $(+-)$ helicity, known as relative luminosity, $R=\mathcal{L}^{++} / \mathcal{L}^{+-}$ $(\approx 1.002)$ :

$$
A_{L L}=\frac{1}{P_{B} P_{Y}} \frac{N^{++}-R N^{+-}}{N^{++}+R N^{+-}}
$$

where $P_{B}$ and $P_{Y}$ are the average beam polarizations for the blue and yellow beam, respectively, and $\mathrm{N}$ is the number of charged pions from the bunch crossings with the same and opposite helicities.

In 2013, nominal beam fills from injection to dump of beams at RHIC lasted eight hours. The PHENIX DAQ system collected data in runs within the fill. Because the prescale of the trigger as well as the polarization values,

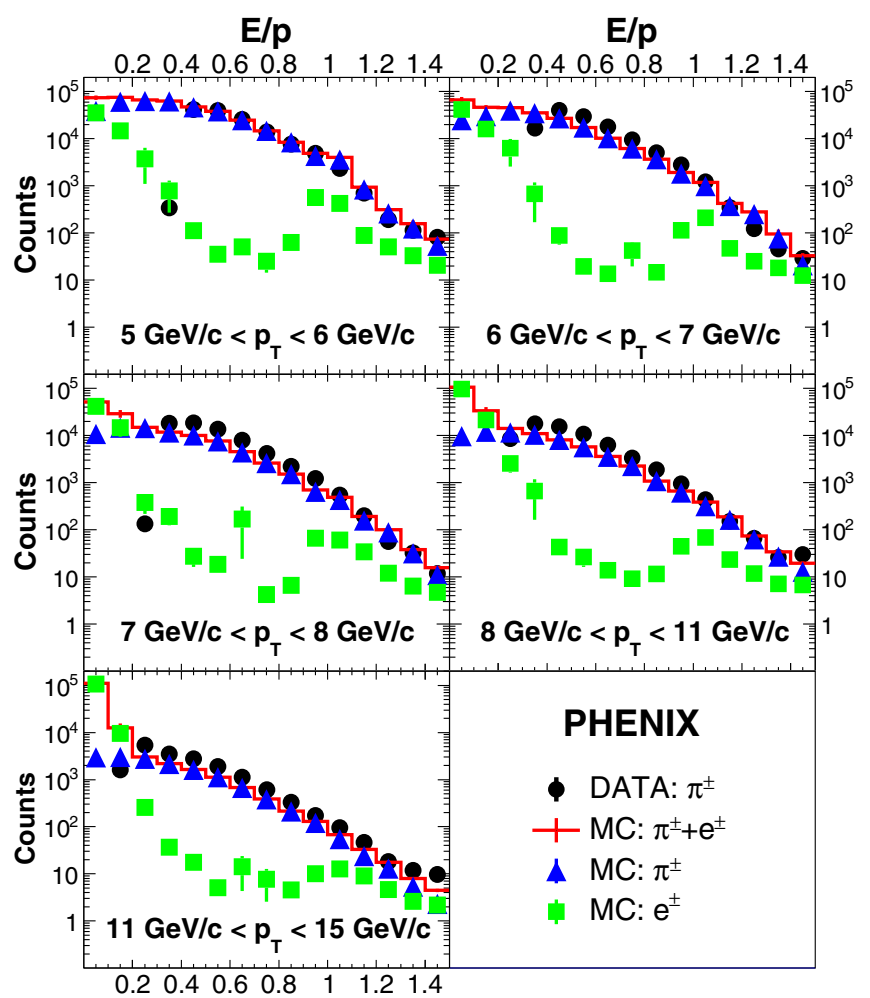

FIG. 4. Energy over momentum ratio for pion candidates in bins of transverse momentum. Reconstructed data (closed [black] circles) are compared to luminosity scaled MC contributions by pions (closed [blue] triangles) and electrons (closed [green] squares) as well as their sum (histogram [red] lines). Due to the minimum energy requirement in the trigger, the data drops at very low values to zero. Note that there are huge tails from true electrons in lower $E / p$ regions. This is because electrons from photon conversion and/or decay in flight are reconstructed with higher transverse momentum and then the measured $E / p$ are lowered. These off-vertex electron backgrounds are eliminated using the $E / p$ cuts.

which were calculated by the initial polarization and the rate of decrease of polarization as a function of time, changed on a run-by-run basis, the analysis is carried out separately for each run. The asymmetries are calculated for each run and each transverse momentum bin and are fit by a constant over all runs. During the 2013 RHIC running period the average beam polarizations $P_{B}$ and $P_{Y}$ were $0.55 \pm 0.02$ and $0.56 \pm 0.02$ for blue and yellow beams, respectively [17].

During the data-taking 16 different spin pattern combinations for the two beams were utilized to minimize systematic effects. These several patterns were found to provide consistent asymmetries, based on T-tests between them, and therefore no systematic uncertainty was assigned due to the different patterns.

To test for other potential systematic effects, the asymmetry calculation is repeated many times with randomized spin patterns for each run. The resulting asymmetry distributions for all iterations peak around zero with a 
Gaussian width given by the statistical uncertainties and the corresponding $\chi^{2} / n$.d.f. distributions of the fits center around unity.

Other systematic uncertainties include a global scale uncertainty of $6.5 \%$ due to the accuracy of the beam polarization determination [17] and the transverse component of the beams, which has been found to be negligible for the double longitudinal spin asymmetries. The uncertainty on the asymmetries based on the relative luminosity extraction is $\delta A_{L L}=3.8 \times 10^{-4}$.

The momentum scale uncertainty of the hadron transverse momentum has also been taken into account, but given the size of the transverse momentum bins used for the asymmetries, bin migration is minimal. The nonpion background has also been considered based on the background yields evaluated by comparing MC with data. The background asymmetry is estimated based on an electron enhanced data sample, which is found to be consistent with zero. The systematic uncertainty from the background asymmetry is evaluated by varying the background fraction after taking into account the evaluated background asymmetry mentioned above. These systematic uncertainties range from $2 \times 10^{-5}$ to $10^{-3}$.

\section{RESULTS}

The resulting final double spin asymmetries are displayed in Fig. 5 as a function of transverse momentum for positive and negative pions and compared to the previously published neutral pions. As can be seen, the results are consistent with the DSSV [5] fit that has considered only the $200 \mathrm{GeV}$ data but not the $510 \mathrm{GeV}$ data. Due to the large statistical uncertainties, the sign of the gluon polarization in the probed $x$ region cannot directly be inferred from the ordering of the asymmetries for the three charges. However, it was found that the present results are consistent with the positive gluon polarization from the global fits. The reason for the comparatively low statistics for charged pions compared to neutral pions is the trigger requirement of having substantial energy deposited in the electromagnetic calorimeter, which happens only for a small fraction of charged pions.

In addition, one can also compare these data to the previously published measurements of charged pions at $\sqrt{s}=200 \mathrm{GeV}$. They are complementary because the hadrons detected at the same transverse momenta but at different center-of-mass energies probe a different momentum fraction region. Therefore, the exact same measurement at higher collision energy of $\sqrt{s}=510 \mathrm{GeV}$ probes a lower value of $x$ than what was possible with the previously published data at $\sqrt{s}=200 \mathrm{GeV}$. While the experimentally measured transverse momentum contains a convolution of $x$ for both partons and the momentum fraction $z$ from the fragmentation process, the variable $x_{T}=2 p_{T} / \sqrt{s}$ can act as a proxy for the $x$ ranges probed. Figure 6 shows

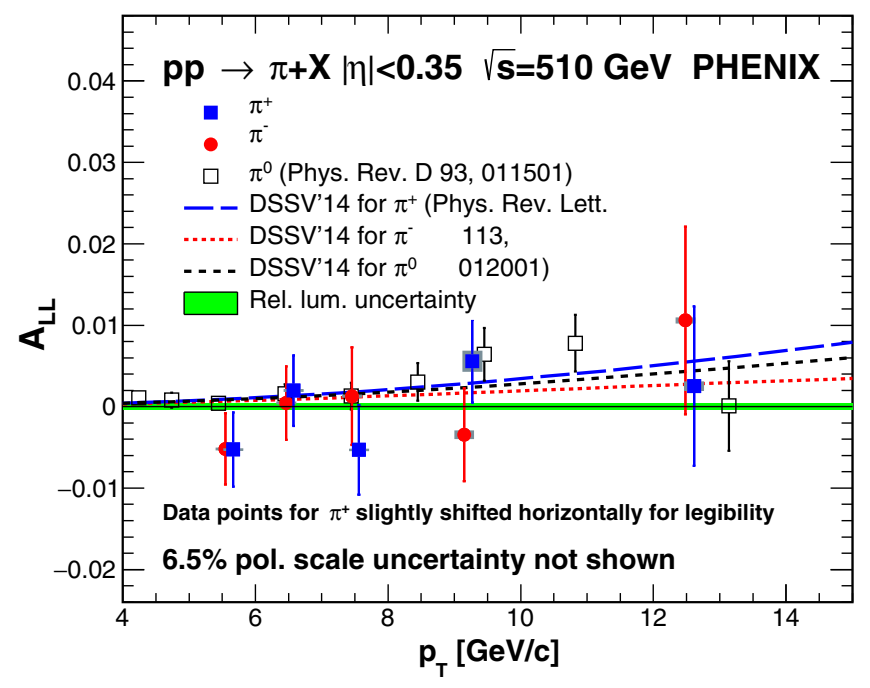

FIG. 5. Double-spin asymmetries $A_{L L}$ as a function of transverse momentum for positive (closed [blue] squares) and negative pions (closed [red] circles), as well as the previously published [7] neutral pions (open [black] squares). The statistical uncertainties of asymmetries and the point-to-point systematic uncertainties from background are represented by the continuous lines and the gray bands, respectively. The expected asymmetries based on the DSSV [5] fit (only from the $200 \mathrm{GeV}$ data but none of the $510 \mathrm{GeV}$ data) are displayed in the indicated line types. The uncertainty bands on the fits are not shown as they affect all charges in similar ways.

the measurements at 200 and $510 \mathrm{GeV}$ and one can see the substantially lower $x_{T}$ reach. Based on PYTHIA [15] simulations of charged pions in the rapidity range and transverse momentum ranges probed in this publication,

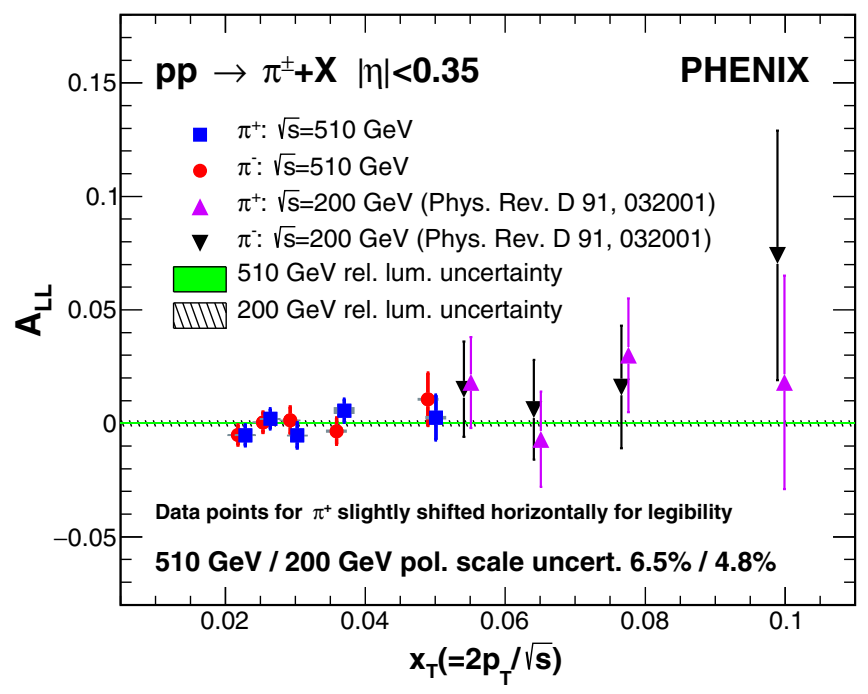

FIG. 6. Double spin asymmetries $A_{L L}$ as a function of $x_{T}=$ $2 p_{T} / \sqrt{s}$ for positive (closed [blue] squares) and negative pions (closed [red] circles) at $\sqrt{s}=510 \mathrm{GeV}$ as well as charged pions at $200 \mathrm{GeV}$ (closed [purple] triangles and closed [black] inverted triangles). The data shown here are tabulated in Table I. 
TABLE I. Charged pion double spin asymmetries $A_{L L}$ in bins of transverse momentum $p_{T}$. The statistical (Stat.) and systematic (Syst.) uncertainties are given in the last two columns.

\begin{tabular}{cccccc}
\hline \hline & $\begin{array}{c}p_{T} \text { bin } \\
{[\mathrm{GeV} / c]}\end{array}$ & $\begin{array}{c}\left\langle p_{T}\right\rangle \\
{[\mathrm{GeV} / c]}\end{array}$ & $\begin{array}{c}A_{L L} \\
{\left[\times 10^{-3}\right]}\end{array}$ & $\begin{array}{c}\text { Stat. } \\
{\left[\times 10^{-3}\right]}\end{array}$ & $\begin{array}{c}\text { Syst. } \\
{\left[\times 10^{-3}\right]}\end{array}$ \\
\hline$\pi^{-}$ & $5-6$ & 5.55 & -5.19 & 4.38 & 0.10 \\
& $6-7$ & 6.47 & 0.45 & 4.51 & 0.10 \\
& $7-8$ & 7.46 & 1.29 & 5.98 & 0.10 \\
& $8-11$ & 9.15 & -3.44 & 5.71 & 0.44 \\
& $11-15$ & 12.48 & 10.60 & 11.52 & 0.30 \\
$\pi^{+}$ & $5-6$ & 5.57 & -5.26 & 4.57 & 0.08 \\
& $6-7$ & 6.48 & 1.97 & 4.34 & 0.09 \\
& $7-8$ & 7.46 & -5.30 & 5.51 & 0.08 \\
& $8-11$ & 9.17 & 5.58 & 5.08 & 1.20 \\
& $11-15$ & 12.51 & 2.52 & 9.78 & 0.49 \\
\hline \hline
\end{tabular}

mean $x$ values of $\approx 0.04-0.09$ can be accessed. Despite the limited statistical precision, this additional information at lower $x$ will improve global fits of the gluon polarization when this data is included. The asymmetries are tabulated in Table I.

\section{SUMMARY}

In summary, PHENIX has measured the charged pion double spin asymmetries at midrapidity $(|\eta|<0.35)$ in longitudinally polarized $p+p$ collisions at $\sqrt{s}=$ $510 \mathrm{GeV}$. These measurements are sensitive to the gluon spin contribution to the total spin of the proton in $x$ range $\approx 0.04-0.09$. The asymmetries are found to be consistent with global fits that have included only $200 \mathrm{GeV}$ RHIC data, and a nonzero, positive gluon polarization in the $x$ region probed by RHIC has been found. In the proposed sPHENIX experiment [18], the hadronic calorimeter will greatly enhance triggering efficiency for charged hadrons and, therefore, significantly improve the statistical precision for charged pion measurements and make such direct evaluation of the gluon spin contribution possible.

\section{ACKNOWLEDGMENTS}

We thank the staff of the Collider-Accelerator and Physics Departments at Brookhaven National Laboratory and the staff of the other PHENIX participating institutions for their vital contributions. We acknowledge support from the Office of Nuclear Physics in the Office of Science of the Department of Energy, the National Science Foundation, Abilene Christian University Research Council, Research Foundation of SUNY, and Dean of the College of Arts and Sciences, Vanderbilt University (U.S.A.), Ministry of Education, Culture, Sports, Science, and Technology and the Japan Society for the Promotion of Science (Japan), Conselho Nacional de Desenvolvimento Científico e Tecnológico and Fundação de Amparo à Pesquisa do Estado de São Paulo (Brazil), Natural Science Foundation of China (People's Republic of China), Croatian Science Foundation and Ministry of Science and Education (Croatia), Ministry of Education, Youth and Sports (Czech Republic), Centre National de la Recherche Scientifique, Commissariat à l'Énergie Atomique, and Institut National de Physique Nucléaire et de Physique des Particules (France), Bundesministerium für Bildung und Forschung, Deutscher Akademischer Austausch Dienst, and Alexander von Humboldt Stiftung (Germany), J. Bolyai Research Scholarship, EFOP, the New National Excellence Program (ÚNKP), NKFIH, and OTKA (Hungary), Department of Atomic Energy and Department of Science and Technology (India), Israel Science Foundation (Israel), Basic Science Research and $\mathrm{SRC}(\mathrm{CENuM})$ Programs through NRF funded by the Ministry of Education and the Ministry of Science and ICT (Korea). Physics Department, Lahore University of Management Sciences (Pakistan), Ministry of Education and Science, Russian Academy of Sciences, Federal Agency of Atomic Energy (Russia), VR and Wallenberg Foundation (Sweden), the U.S. Civilian Research and Development Foundation for the Independent States of the Former Soviet Union, the Hungarian American Enterprise Scholarship Fund, the U.S.-Hungarian Fulbright Foundation, and the U.S.-Israel Binational Science Foundation.
[1] J. Ashman et al. (European Muon Collaboration), A measurement of the spin asymmetry and determination of the structure function $g_{1}$ in deep inelastic muon-proton scattering, Phys. Lett. B 206, 364 (1988).

[2] R. L. Jaffe and A. Manohar, The G(1) problem: Fact and fantasy on the spin of the proton, Nucl. Phys. B337, 509 (1990).
[3] L. Adamczyk et al. (STAR Collaboration), Precision Measurement of the Longitudinal Double-spin Asymmetry for Inclusive Jet Production in Polarized Proton Collisions at $\sqrt{s}=200 \mathrm{GeV}$, Phys. Rev. Lett. 115, 092002 (2015).

[4] A. Adare et al. (PHENIX Collaboration), Inclusive double-helicity asymmetries in neutral-pion and eta-meson 
production in $\vec{p}+\vec{p}$ collisions at $\sqrt{s}=200 \mathrm{GeV}$, Phys. Rev. D 90, 012007 (2014).

[5] D. de Florian, R. Sassot, M. Stratmann, and W. Vogelsang, Evidence for Polarization of Gluons in the Proton, Phys. Rev. Lett. 113, 012001 (2014).

[6] E. R. Nocera, R. D. Ball, S. Forte, G. Ridolfi, and J. Rojo (NNPDF Collaboration), A first unbiased global determination of polarized PDFs and their uncertainties, Nucl. Phys. B887, 276 (2014).

[7] A. Adare et al. (PHENIX Collaboration), Inclusive cross section and double-helicity asymmetry for $\pi^{0}$ production at midrapidity in $p+p$ collisions at $\sqrt{s}=510 \mathrm{GeV}$, Phys. Rev. D 93, 011501 (2016).

[8] J. Adam et al. (STAR Collaboration), Longitudinal doublespin asymmetries for dijet production at intermediate pseudorapidity in polarized $p p$ collisions at $\sqrt{s}=200 \mathrm{GeV}$, Phys. Rev. D 98, 032011 (2018).

[9] J. Adam et al. (STAR Collaboration), Longitudinal doublespin asymmetries for $\pi^{0} \mathrm{~s}$ in the forward direction for $510 \mathrm{GeV}$ polarized $p p$ collisions, Phys. Rev. D 98, 032013 (2018).

[10] J. Adam et al. (STAR Collaboration), Longitudinal doublespin asymmetry for inclusive jet and dijet production in pp collisions at $\sqrt{s}=510 \mathrm{GeV}$, Phys. Rev. D 100, 052005 (2019).

[11] L. Adamczyk et al. (STAR Collaboration), Measurement of the cross section and longitudinal double-spin asymmetry for di-jet production in polarized $p p$ collisions at $\sqrt{s}=200 \mathrm{GeV}$, Phys. Rev. D 95, 071103 (2017).

[12] D. de Florian, R. Sassot, M. Epele, R. J. Hernández-Pinto, and M. Stratmann, Parton-to-pion fragmentation reloaded, Phys. Rev. D 91, 014035 (2015).

[13] A. Adare et al. (PHENIX Collaboration), Charged-pion cross sections and double-helicity asymmetries in polarized $\mathrm{p}+\mathrm{p}$ collisions at $\sqrt{s}=200 \mathrm{GeV}$, Phys. Rev. D 91, 032001 (2015).

[14] K. Adcox et al. (PHENIX Collaboration), PHENIX detector overview, Nucl. Instrum. Methods Phys. Res., Sect. A 499, 469 (2003).

[15] T. Sjöstrand, S. Mrenna, and P.Z. Skands, PYTHIA6.4 physics and manual, J. High Energy Phys. 05 (2006) 026.

[16] R. Brun, F. Bruyant, F. Carminati, S. Giani, M. Maire, A. McPherson, G. Patrick, and L. Urban, GEANT detector description and simulation tool, Reports No. CERN-W5013, No. CERN-W-5013, No. W5013, and No. W-5013, 1994, https://cds.cern.ch/record/1082634.

[17] W. D. Schmidke (The RHIC Polarimetry Group), RHIC polarization for runs 9-17 (2018), https://technotes.bnl.gov/ Home/ViewTechNote/209057.

[18] sPHENIX Collaboration, Medium-energy nuclear physics measurements with the sPHENIX barrel (2017), https://indico.bnl.gov/event/3866/attachments/10441/12744/ medium-energy-nuclear.pdf. 